\title{
Fatal Meningoencephalomyelitis due to the Tick-borne Encephalitis Virus: The First Detailed Neurological Observation in a Japanese Patient from the Central Part of Hokkaido Island
}

\author{
Yasutaka Tajima, Hiroaki Yaguchi and Yasunori Mito
}

\begin{abstract}
:
To date, the only instance of tick-borne encephalitis (TBE) in Japan was reported from the southern part of Hokkaido Island in 1993; no other cases have been reported since then. We herein report the first case of TBE reported in the central part of Hokkaido Island, and describe the fatal clinical course of a patient who presented with meningoencephalomyelitis, which partly involved the nerve root. Magnetic resonance imaging (MRI) of the patient's cranium and spine revealed characteristic central nervous system involvement. Our case report is extremely relevant to efforts to protect public health and for precautions against TBE pandemics.
\end{abstract}

Key words: tick-borne encephalitis, magnetic resonance imaging, meningoencephalomyelitis

(Intern Med 57: 873-876, 2018)

(DOI: 10.2169/internalmedicine.8437-16)

\section{Introduction}

Tick-borne encephalitis (TBE) is widely endemic from Europe to the far-eastern part of Russia. The causative pathogen is the tick-borne encephalitis virus (TBEV), which is classified into the European, Siberian and Far-Eastern subtypes (1). To date, the only case of TBE in Japan was reported in 1993, from the southern part of Hokkaido Island $(2,3)$. A detailed field investigation demonstrated that a type of TBEV, the Russian spring-summer encephalitis virus, was widely distributed in that region (3). We herein report the first case of TBE in the central part of Hokkaido Island, which was reported from near Sapporo City, and describe the fatal clinical course of the patient who presented with meningoencephalomyelitis, which partly involved the nerve root. Our report is significant because it involves a neuroinfection that is extremely rare in Japan. It is relevant because it concerns the Japanese public health system and how the Japanese population can be protected against arthropod-transmitted infections.

\section{Case Report}

A 44-year-old man, with no remarkable medical history, presented with muscle weakness in his left leg and difficulty in walking. He reported that he had visited a forest in a suburb of Sapporo City to take photographs 11 days prior to his admission. During his visit, he received a tick bite, which was subsequently treated via tissue resection and a short course of antibiotics. The man reportedly felt discomfort in his abdomen and thighs 8 days after the tick bite. His body temperature rose to $39^{\circ} \mathrm{C}$ and he became aware of arthralgia and muscle pain the following day. As mentioned, the man also noticed muscle weakness in his left leg and reported difficulty in walking. He was therefore referred to our department. A neurological examination revealed that the patient was alert and showed no indication of meningeal irritation. There was no evidence of cranial nerve or cerebellar involvement. He did not complain of muscle weakness in his upper extremities. The deep tendon reflexes (DTRs) of the patient's upper extremities were also normal. In contrast, his left leg was hypotonic and exhibited weakness (muscle 


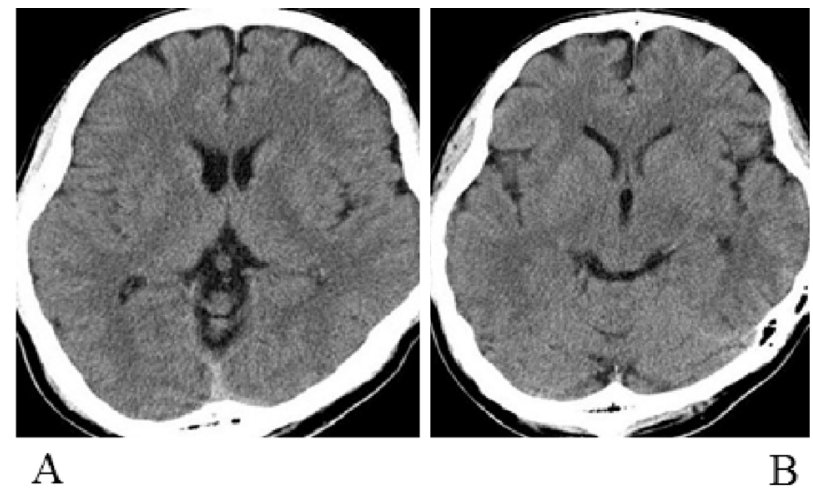

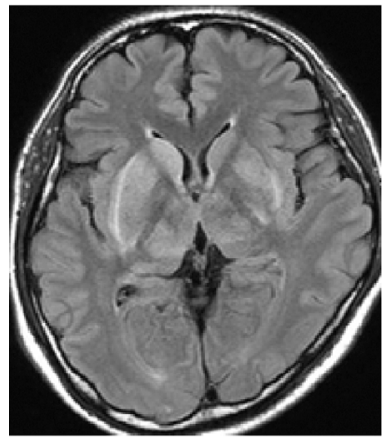

$\mathrm{C}$

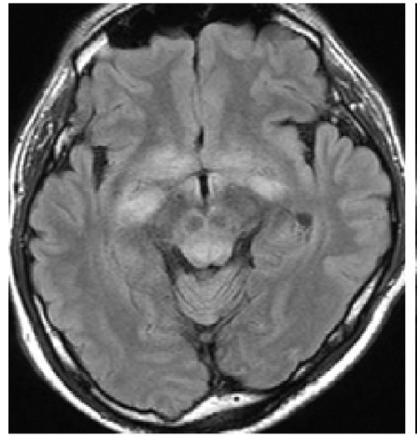

D

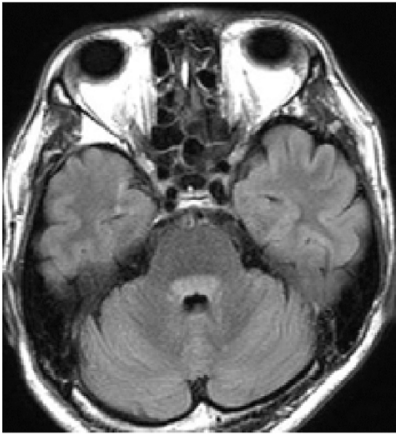

$\mathrm{E}$

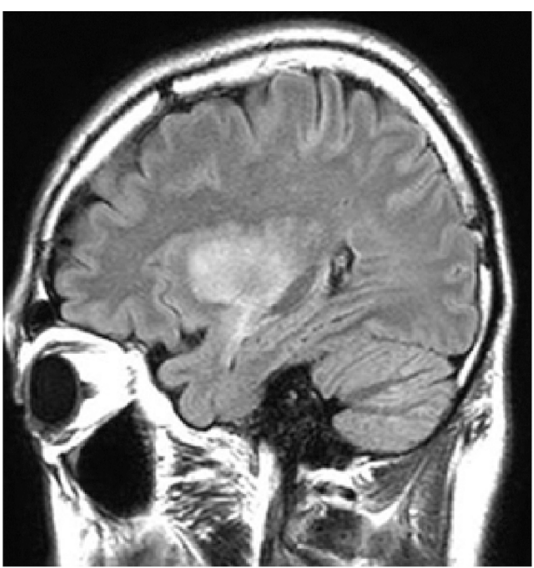

$\mathrm{F}$

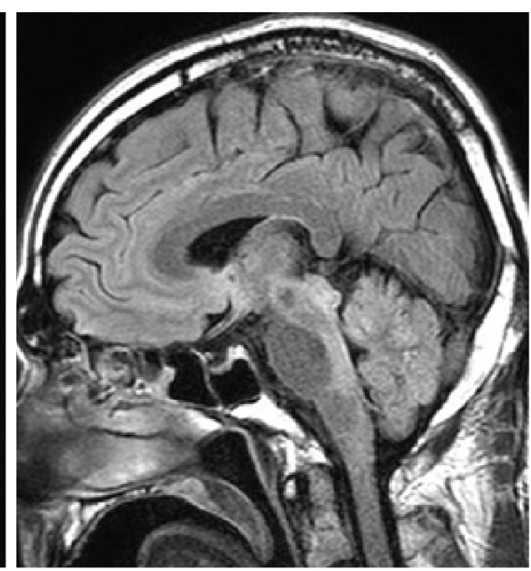

G

Figure 1. The Cranial CT and MRI findings. A, B: Cranial CT on admission revealed no abnormalities. C, D, and E: Axial FLAIR MRI demonstrated areas of high signal intensity in the basal ganglia (C), bilateral thalamus, midbrain (D), and pontine tegmentum (E). F, G: Sagittal FLAIR MRI demonstrated the vast involvement of the thalamus and the dorsal part of the brain stem.

manual testing: 3). Additionally, the patient's left-sided patellar tendon reflex (PTR) was hypoactive and his left Achilles tendon reflex (ATR) was diminished; both the right PTR and right ATR were well preserved. Pathological reflexes could not be elicited bilaterally, and the patient could stand with assistance but could not walk. The patient experienced slight urinary disturbance and discomfort below his abdomen. Electrophysiological investigations revealed that the conduction velocities and compound muscle action potentials were normal; however, left tibial nerve stimulation did not fully evoke F waves. The latter result suggested nerve root or anterior horn involvement. Cranial computed tomography ( CT ) demonstrated normal findings
(Fig. 1A and B), while T2-weighted spinal magnetic resonance imaging (MRI) indicated a longitudinal area of high signal intensity in the center of the patient's spinal cord, particularly in the grey matter (Fig. 2B, D and E). Gadolinium-enhanced MRI did not demonstrate any enhancement of the spinal cord, but part of the cauda equina showed enhancement (Fig. 2C). Cerebrospinal fluid (CSF) examinations revealed pleocytosis $\left(337 / \mathrm{mm}^{3}\right.$ : mononuclear cells, 216/ $\mathrm{mm}^{3}$; polynuclear cells, $121 / \mathrm{mm}^{3}$ ) and an elevated protein content of $230 \mathrm{mg} / \mathrm{dL}$. The CSF glucose level was $47 \mathrm{mg} / \mathrm{dL}$, and a cytological examination was negative without bacterial culture. Based on these findings, we diagnosed the patient with meningomyeloradiculopathy due to Lyme 


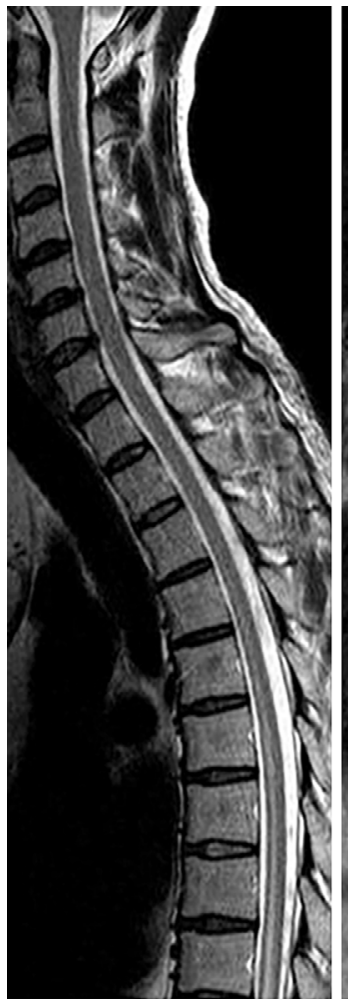

A

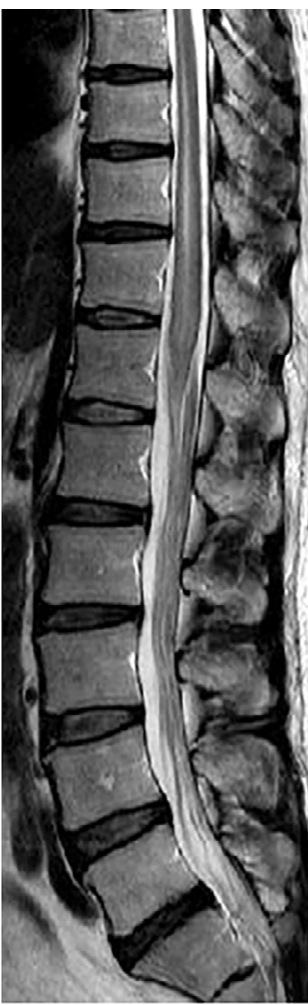

$\mathrm{B}$
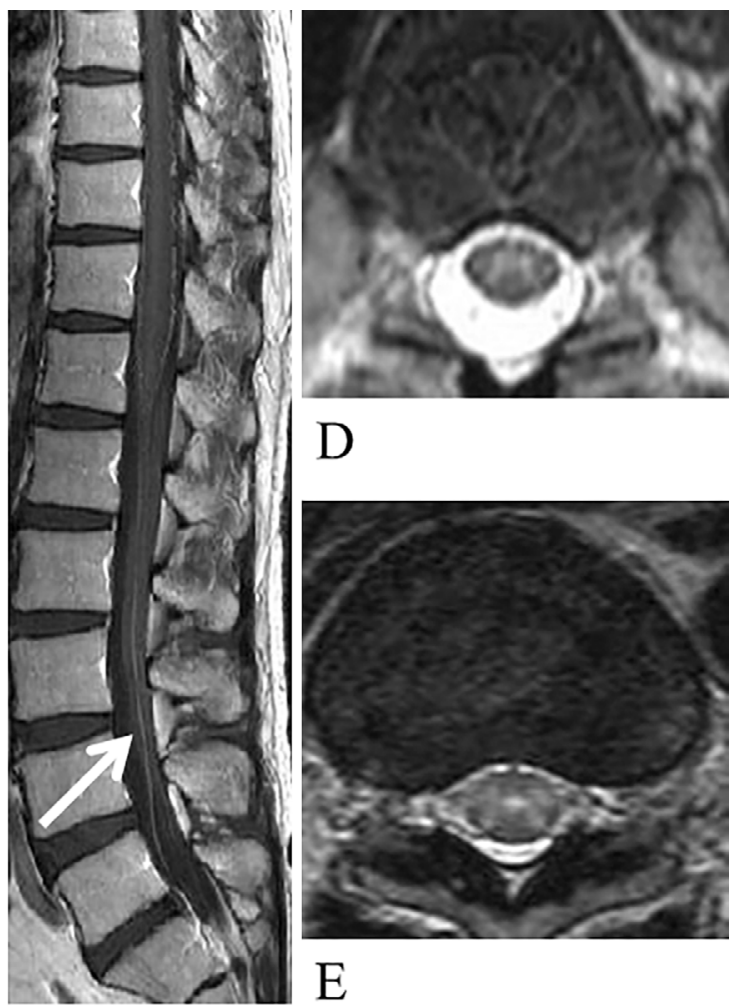

$\mathrm{D}$

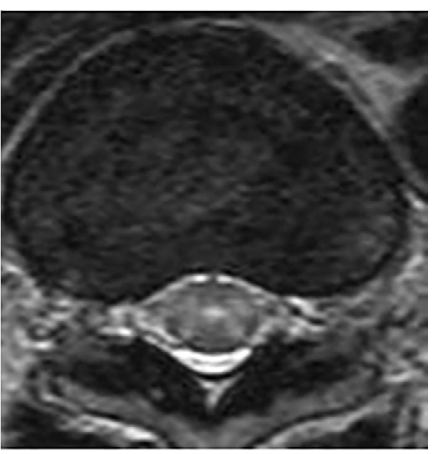

$\mathrm{E}$

$\mathrm{C}$

Figure 2. Spinal MRI. A, B: T2-weighted MRI of the spinal cord demonstrated areas of high signal intensity in the central region of the spinal cord, from $\mathrm{T} 3$ to the end of the spinal cord; the conus exhibited slight cord swelling. C: Part of the cauda equina showed gadolinium enhancement (arrow). D, E: T2-weighted axial MRI image revealed an area of high signal intensity in the grey matter of the spinal cord with slight cord swelling.

disease and prescribed ceftriaxone ( $2 \mathrm{~g} / \mathrm{day}$, intravenously) and pulse steroid therapy. Despite these treatments, the weakness in both legs worsened and the bilateral PTRs and ATRs were found to have decreased on the day after admission. T2-weighted MRI of the patient's spinal cord revealed a longitudinal area of high signal intensity below T3 and the absence of cervical lesions (Fig. 2A). He suddenly developed respiratory failure and required intubation with mechanical ventilation on the 2 nd day after admission. The patient's consciousness was not impaired, but the DTRs of all four extremities were diminished and weakness of his neck muscles and bilateral facial muscles became evident. Because we suspected that his clinical condition was severely affected by immune-mediated radiculoneuropathy, several courses of plasma exchange were implemented. Unfortunately, this additional treatment did not have any beneficial effects, and the patient developed generalized convulsions the 3rd day after admission. On that occasion, cranial MRI with fluid attenuated inversion recovery (FLAIR) demonstrated a symmetrical area of high signal intensity involving the thalamus, basal ganglia, pontine tegmentum, and the dorsal part of the medulla (Fig. 1C-G). Despite intensive treatment, the patient was pronounced brain dead 10 days later. At that time, we could not evoke any brain stem reflexes, and confirmed the flattening of the electroencephalo- gram and the loss of auditory-evoked responses. Subsequent tests of the patient's serum for anti-Borrelia burgdorferi $\operatorname{IgG}$ and $\operatorname{IgM}$ antibodies were negative, as were tests for GM1 and GQ1b anti-ganglioside antibodies. Conversely, the serum levels of antibodies against flavivirus were highly elevated; thus, the diagnosis of TBE was confirmed. Finally, the patient was declared dead on the 20th day after admission.

\section{Discussion}

The patient was a resident of a suburb of Sapporo City in the central region of Hokkaido Island; this district is at least $350 \mathrm{~km}$ from the area in which the first case of TBEV in Japan was recorded 23 years ago $(2,3)$. Additional field investigations of the area where this patient contracted the virus are necessary to determine the distribution of the virus. It is also possible that TBEV could be more widely spread across Hokkaido Island than previously estimated; thus, future outbreaks of TBEV cannot be ruled out. Taken together, these points suggest that TBEV represents a major concern for the Japanese public health system, specifically in relation to controlling and taking precautions against arthropodtransmitted diseases. In our case, the neurological symptoms began with lower spinal cord involvement, which presented 
as bilateral leg weakness, and progressed upward along the spinal cord to the T3 level. Within the spinal cord, the grey matter exhibited high-intensity signals on T2-weighted MRI, indicating viral neurotropism (4). Although cranial CT at the initial examination did not demonstrate any abnormal findings, the patient developed repeated convulsions. Subsequent cranial FLAIR MRI demonstrated lesions with symmetric areas of high signal intensity involving the bilateral thalamus, basal ganglia, and brainstem. These characteristics along with the widely distributed central nervous system abnormalities on MRI were consistent with those of previous studies $(5,6)$. Thus, even in Japan, TBE should be considered when patients with encephalomyelitis exhibit similar MRI findings. Although radicular involvement, such as that observed in this case, is a very rare symptom of TBE, its possibility should never be overlooked in TBE patients $(7,8)$. The mortality rate associated with TBE is estimated to be $1 \%(1,6)$, and our patient was eventually pronounced brain dead. The development of useful vaccinations and effective antiflavivirus drugs is necessary to help prevent and treat this devastating disease.

The authors state that they have no Conflict of Interest (COI).

\section{Acknowledgement}

The authors thank Dr. Kentaroh Yoshi (Department of Veterinary Medicine, Hokkaido University) for measuring the viral antibodies and Dr. Annei Moriuchi (Department of Dermatology, Sapporo City General Hospital) for providing us with scientific suggestions.

\section{References}

1. Lindquist L, Vapalahti O. Tick-borne encephalitis. Lancet 371: 1861-1871, 2008.

2. Takezawa C, Sato T, Mizutani Y, Abe S, Morita K. Russian spring-summer encephalitis. A case report. Neurol Med 43: 251255, 1995 (in Japanese, Abstract in English).

3. Takashima I, Morita K, Chiba M, et al. A case of tick-borne encephalitis in Japan and isolation of the virus. J Clin Microbiol 35: 1943-1947, 1997.

4. Beer S, Brune N, Kesselring J. Detection of anterior horn lesions by MRI in central European tick-borne encephalomyelitis. J Neurol 246: 1169-1171, 1999.

5. Bender A, Schulte-Altedorneburg G, Walther EU, Pfister H-W. Sever tick borne encephalitis with simultaneous brain stem, bithalamic, and spinal cord involvement documented by MRI. J Neurol Neurosurg Psychiatry 76: 135-137, 2005.

6. Valdueza JM, Weber JR, Harms L, Bock A. Sever tick borne encephalomyelitis after tick bite and passive immunization. J Neurol Neurosurg Psychiatry 60: 593-594, 1996.

7. Fauser S, Stich O, Rauser S. Unusual case of tick borne encephalitis with isolated myeloradiculitis. J Neurol Neurosurg Psychiatry 78: 909-910, 2007.

8. Racz A, Schaller G, Lunkenheimer J, et al. Isolated meningomyeloradiculitis following infection with tick borne encephalitis virus. Clin Neurol Neurosurg 114: 1283-1286, 2012.

The Internal Medicine is an Open Access article distributed under the Creative Commons Attribution-NonCommercial-NoDerivatives 4.0 International License. To view the details of this license, please visit (https://creativecommons.org/licenses/ by-nc-nd/4.0/).

(C) 2018 The Japanese Society of Internal Medicine Intern Med 57: 873-876, 2018 